Meta

Journal des traducteurs

Translators' Journal

\title{
Le facteur local comme levier d'une traductologie pragmatique
}

\section{Nicolas Froeliger}

Volume 55, numéro 4, décembre 2010

De la localisation à la délocalisation - le facteur local en traduction

From Localization to Delocalization - The Local Factor in Translation

URI : https://id.erudit.org/iderudit/045683ar

DOI : https://doi.org/10.7202/045683ar

Aller au sommaire du numéro

Éditeur(s)

Les Presses de l'Université de Montréal

ISSN

0026-0452 (imprimé)

1492-1421 (numérique)

Découvrir la revue

Citer cet article

Froeliger, N. (2010). Le facteur local comme levier d'une traductologie pragmatique. Meta, 55(4), 642-660. https://doi.org/10.7202/045683ar

\section{Résumé de l'article}

Où se manifeste le facteur local en traduction pragmatique, avec quels enjeux et quels horizons théoriques ? Abordée à partir d'exemples empruntés à la technique, à l'économie et à la presse, cette question débouche sur un paradoxe traductologique : celui de réunir artificiellement, sur l'importance de traduire non pas ce que le texte dit, mais ce qu'il fait, des auteurs en totale contradiction, et dont les seuls points communs semblent être une prise de distance par rapport à la linguistique et une réflexion fondée sur la traduction de la Bible. Tenter de surmonter ce paradoxe afin de produire, à partir du même facteur local, une recherche pertinente en pragmatique conduit à se demander si le problème n'est pas dans le choix des textes de référence, ainsi que sur la possibilité et l'intérêt d'une émancipation de la traductologie pragmatique par rapport aux théories plus littéraires ou se voulant généralisantes. Cette démarche permet in fine d'isoler un trait que nous pensons décisif en traduction pragmatique : c'est lorsqu'il y a défaut (inaperçu) d'identité entre source et cible que l'on peut dire qu'il y a véritablement traduction.
Ce document est protégé par la loi sur le droit d'auteur. L’utilisation des services d'Érudit (y compris la reproduction) est assujettie à sa politique d'utilisation que vous pouvez consulter en ligne.

https://apropos.erudit.org/fr/usagers/politique-dutilisation/ 


\title{
Le facteur local comme levier d'une traductologie pragmatique
}

\author{
NICOLAS FROELIGER* \\ Université Paris Diderot - Paris 7, Paris, France \\ nf@eila.univ-paris-diderot.fr
}

\begin{abstract}
RÉSUMÉ
Où se manifeste le facteur local en traduction pragmatique, avec quels enjeux et quels horizons théoriques? Abordée à partir d'exemples empruntés à la technique, à l'économie et à la presse, cette question débouche sur un paradoxe traductologique: celui de réunir artificiellement, sur l'importance de traduire non pas ce que le texte dit, mais ce qu'il fait, des auteurs en totale contradiction, et dont les seuls points communs semblent être une prise de distance par rapport à la linguistique et une réflexion fondée sur la traduction de la Bible. Tenter de surmonter ce paradoxe afin de produire, à partir du même facteur local, une recherche pertinente en pragmatique conduit à se demander si le problème n'est pas dans le choix des textes de référence, ainsi que sur la possibilité et l'intérêt d'une émancipation de la traductologie pragmatique par rapport aux théories plus littéraires ou se voulant généralisantes. Cette démarche permet in fine d'isoler un trait que nous pensons décisif en traduction pragmatique: c'est lorsqu'il y a défaut (inaperçu) d'identité entre source et cible que l'on peut dire qu'il y a véritablement traduction.
\end{abstract}

\begin{abstract}
Where will the local factor be encountered in pragmatic translation? What is at stake there and which theories do those phenomena relate to? This question is first considered using examples from technical, business or journalistic texts. It leads, however, to a paradox, one that artificially agrees on the importance of translating the thrust of the text, not just what it says, and thus unites authors that disagree on nearly everything else and whose sole common points are a certain reluctance toward linguistic approaches and the use of the Bible as a touchstone for their reflections. Trying to overcome this paradox to produce research that is useful and operative in pragmatic translation leads to two questions. First, does the problem lie in the choice of reference texts? Second, is it possible and worthwhile to emancipate pragmatic translation studies from other theories that are more inclusive or specifically grounded in literary texts? This approach ultimately reveals a feature we consider decisive in pragmatic translation: the core of the translating activity emerges in those rare places where identity between source and target texts is absent, albeit imperceptibly.
\end{abstract}

\section{MOTS-CLÉS/KEYWORDS}

traductologie pragmatique, facteur local, traduction technique, traduction littéraire, topologie

pragmatic translation studies, local factor, technical translation, literary translation, topology

La localisation constitue-t-elle un phénomène nouveau? Assurément si on la définit à la fois comme une intervention portant sur le code informatique et sur le texte. Indéniablement si l'on considère les dénominations professionnelles: la spécialité de localisateur (ou localiseur) existe aujourd'hui de plein droit tout en ressortissant bel 
et bien, comme celle de rédacteur technique, aux métiers de la traduction. Et pourtant... ? Est-ce que l'on n'a pas toujours localisé, c'est-à-dire intégré les données nécessaires à la réception du texte d'arrivée, depuis que l'on traduit? Ce que l'on appelle, au sens étroit, localisation n'est-il pas une simple extension à des supports nouveaux de pratiques aussi anciennes que la traduction tout court, et subsumables à cette dernière: adaptation à un public, à une époque, à un besoin donnés? Lorsque saint Jérôme modifie la prononciation d'un mot de passe hébreu (shibbolet devenant sebboleth, Livre des Juges, 12, 5-6) pour le rendre prononçable en latin (ce qui n'est pas anodin puisqu'une prononciation autre - sibbolet ou sebbolet - valait arrêt de mort $^{1}$ ), sa démarche est-elle au fond différente de celle du localisateur d'aujourd'hui qui adapte à la législation du pays destinataire les mentions légales accompagnant le texte qu'il doit rendre ou qui modifie le code informatique de ce même texte en fonction des normes du pays d'arrivée? Qui a raison? Les tenants de la nouveauté ou ceux de la continuité? Les uns et les autres, évidemment, dans leurs logiques respectives. La question, pour la traductologie, est plutôt de savoir de quelle manière les amener à avoir raison ensemble: comment une réflexion large sur le facteur local, partant de ce qui constitue aujourd'hui la localisation (et nous nous référons pour cela à l'article de Cancio et Belmonte [2010], dans le présent numéro thématique), peut-elle nous aider à articuler une réflexion en traductologie pragmatique? C'est pour cette raison que nous laisserons de côté les aspects informatiques, en les considérant seulement comme une extension non textuelle de pratiques qui commencent avec le texte. Cette problématique se divisera en deux parties. La première est éminemment concrète: il s'agira de nous interroger sur différentes manières de traiter, en traduction pragmatique, le facteur local, afin d'esquisser quelques voisinages théoriques. La seconde est plus globale. Elle cherchera, à partir de ces réflexions sur le facteur local, à isoler et situer la traduction pragmatique dans un paysage traductologique général. La question du lieu nous servira donc à la fois pour identifier des points pertinents pour la réflexion et pour tenter de replacer la traductologie pragmatique dans son environnement.

\section{Les lieux du local}

Quels sont, dans un premier temps, les lieux du local en traduction pragmatique? En d'autres termes, dans quelles circonstances un traducteur va-t-il s'écarter d'une simple transposition des composantes linguistiques du texte de départ? Nous allons, dans cette partie, poser cette question à partir d'exemples tirés de l'expérience (la nôtre et celle de collègues et étudiants avec lesquels nous avons partagé cette réflexion), pour tenter de parvenir à un ensemble de lieux communs, au double sens de ce terme - le banal et l'aristotélicien (un point d'accord partagé) -, car nous n'entendons pas, à ce stade, dire quelque chose de nouveau, mais plutôt relier une pratique à des travaux déjà bien connus. Nous partirons pour cela des domaines où l'intervention du facteur local semble a priori la plus improbable (la technique), pour aller vers ceux où elle est plus attendue (les références culturelles en traduction journalistique). 


\subsection{L’universalité de la technique à l'épreuve du local}

On pourrait penser que la technique est le lieu de l'unicité de chaque spécialité. Les lois scientifiques sur lesquelles elle repose ne se jouent-elles pas a priori de toutes les frontières - ce qui en ferait par exemple l'opposé de la traduction juridique, où la composante territoriale constitue un élément décisif? Si, bien sûr. Et pourtant, l'expérience montre que l'aspect géographique a, même dans ce bastion de l'universel, un rôle à jouer. C'est donc, comme toujours, aux exceptions, à tout ce qui est susceptible de contredire l'intuition, qu'il faut s'intéresser.

On constate d'abord que le local intervient dans la sphère terminologique. Ainsi, les grandeurs et outils mathématiques dont l'appellation est dérivée d'un nom propre (généralement celui d'un inventeur: Siemens, Volta, Watt, en électricité, par exemple) ne se traduisent pas toujours littéralement. Dans la famille des coefficients sans dimension, ou adimensionnels (c'est-à-dire valables dans toutes les dimensions), ce que les anglophones appellent Eckert Number $(\mathrm{Ec})$, et qui sert à caractériser l'énergie cinétique d'un fluide, se dit en français nombre de Dulong $(\mathrm{Du})$, tandis que le Stanton Number (St), qui décrit le transfert thermique ou le transfert de masse, équivaut au nombre de Margoulis $(\mathrm{Mr})^{2}$. Pourquoi ? Parce que leur découverte, comme d'ailleurs l'invention du gramophone ou, beaucoup plus près de nous, la description du virus du sida, a eu lieu simultanément dans des pays différents en situation de concurrence scientifique. Les divergences d'appellation, piège conséquent pour le traducteur, sont donc l'expression d'une rivalité historique entre puissances.

Le facteur local intervient également avec la nécessaire adaptation des textes techniques à un marché cible, comme le montre, dans le tableau 1, l'extrait d'un texte sur les moyens de lutter contre le bruit dans le bâtiment.

TABLEAU 1

\section{Effacer l'inconnu}

Double pane and/or storm windows reduce sound transmission. Weather stripping helps. Windows facing exterior noise sources should be small and as few as possible. Double-hung windows should be able to be tightly closed.
Des fenêtres à double vitrage ou des contrefenêtres réduisent la transmission du son. Les fenêtres faisant face à des sources sonores extérieures doivent idéalement être petites et peu nombreuses. Enfin, il doit être possible de fermer correctement les fenêtres à guillotine.

(CertainTeed Corporation 2002: 1133)

S’agissant d'un texte américain à traduire pour un public français, la phrase en caractères gras a tout simplement été omise, car la notion de fenêtre coupe-bise (terme canadien-français pour l'anglais weather stripping) n'existe pas dans la terre francophone de l'Ancien Monde. Une traduction linguistiquement correcte aurait donc été factuellement fautive. À moins, éventuellement, de préciser la zone géographique concernée: "Aux États-Unis et au Canada, les dispositifs coupe-bise contribuent également à cet effet.» L'efficacité du message (ne faire figurer que des informations pertinentes pour le lecteur) y aurait néanmoins perdu ce que le souci d'exhaustivité (restituer rigoureusement chaque élément de sens) y aurait gagné, et il n'est pas certain que ce jeu soit à somme nulle.

Inversement, on reprendra en français (et au mépris conscient, pour une fois, du code typographique, ce livre de chevet du traducteur) un sigle étranger si celui-ci est majoritairement employé dans les pays francophones: 
TABLEAU 2

\section{Accepter l'étranger}

A new single number rating for sound absorption that will be replacing the NRC over the next several years is the sound absorption average (SAA). This is the average of the sound absorption coefficients of a material from 200 through $2500 \mathrm{~Hz}$ inclusive.
Un nouvel indice pour mesurer l'absorption sonore vient de voir le jour et remplacera le coefficient de réduction du bruit dans les années à venir. Il s'agit du coefficient d'absorption moyen (SAA), qui calcule la moyenne des coefficients d'absorption d'un matériau de 200 à $2500 \mathrm{~Hz}$ inclus.

(CertainTeed Corporation 2002: $11^{3}$ )

Et tout traducteur un tant soit peu expérimenté sait bien que la décision de convertir (ou non) les unités de mesure dépend non seulement des pays, mais aussi des publics concernés. Ainsi, la taille des écrans de visualisation (qui est toujours, en anglais, indiquée en pouces) sera, en français, convertie en centimètres pour les téléviseurs, et restera en pouces pour les terminaux d'ordinateur. Cette différence va d'ailleurs rapidement poser des problèmes de choix et de cohérence vu la convergence rapide des deux domaines ${ }^{4} .$.

Même la description des phénomènes physiques peut être tributaire du lieu. Prenons un texte (allemand) décrivant un dispositif de dépoussiérage utilisé dans les chaînes de mise en peinture du secteur automobile. Ces machines captent la poussière présente ou susceptible de se déposer sur les surfaces externes des carrosseries au moyen de rouleaux en plumes d'émeu, qui sont de surcroît chargées électrostatiquement. S'adressant à des ingénieurs de l'automobile et à des carrossiers, l'auteur juge nécessaire de rappeler les principes de l'électrostatique. Voici le début de son explication, extraite d'un document interne que nous avons eu à traduire ${ }^{5}$ :

(1) Elektrostatische Aufladung entsteht immer dann, wenn die Oberflächen zweier verschiedener Werkstoffe, gleicher Temperatur, voneinander getrennt werden. Beim mechanischen Kontakt dieser Stoffe entsteht in der Grenzschicht ein Elektronenaustausch, durch den das thermodynamische Gleichgewicht wiederhergestellt wird.

S'agissant d'un principe de base de la physique, il est assez simple de traduire ce que dit ce texte en utilisant peu ou prou le même type de formulation. C'est la proposition de certains de nos étudiants.

TABLEAU 3

\section{Une traduction linguistiquement et factuellement correcte}

Une charge électrostatique se crée dès lors que les surfaces de deux matériaux différents, à la même température, sont séparées l'une de l'autre. L'interaction mécanique de ces matériaux produit, au niveau de leur couche limite, un échange d'électrons qui rétablit l'équilibre thermodynamique.
Dès lors: traduction habile (mais en fait inutile) du immer qui n'avait pas sa place dans l'original allemand: lorsqu'on énonce une règle scientifique générale, on n'a pas à préciser qu'elle est toujours vraie... Ce dès lors est donc une traduction maniériste, fidèle, dans un domaine que Berman méprise, aux préceptes prônés par Berman (1999) (d'après les observations des étudiants).

Cette traduction est correcte. Pour un ingénieur français de France, elle est néanmoins inattendue, car le phénomène en question n'est pas enseigné ou expliqué 
de la même manière dans ce pays: on ne met pas l'accent sur la séparation mais sur le frottement. Et pourtant, la charge électrostatique produite sera la même, puisque le frottement n'est jamais qu'une séparation répétée. Un rapide aperçu des sites francophones sur la question montre (fin 2009) qu'une seule page Internet reprend l'idée de séparation, et qu'elle est, comme par hasard, traduite de l'allemand...

Une traduction totalement consciencieuse (mais à la vérité, ce degré de conscience professionnelle est rarement accessible aux traducteurs professionnels, faute de temps) aurait abouti à adapter cette explication en fonction du facteur local. C'est ce qu'a suggéré une autre de nos étudiantes (tableau 4):

\section{TABLEAU 4}

\section{Une traduction localisée}

Le frottement répété de deux matériaux différents, à la même température produit une charge électrostatique. En effet, l'interaction mécanique de ces matériaux se traduit, au niveau de leur couche limite, par un échange d'électrons qui rétablit l'équilibre thermodynamique.
Se traduit: autre maniérisme de traducteur (d'après les observations des étudiants).

Est-on, ici, dans un problème de terminologie ou de métaphores de référence? Nous ne sommes pas suffisamment versés en terminologie, justement, pour pouvoir répondre à cette question de manière décisive. En tout cas, on est assurément face à un problème majeur du discours scientifique, qui est aussi construit de manière à imposer une vision du domaine considéré - Thomas Kuhn dirait un paradigme - avec à la clé un premier risque d'impérialisme culturel. Une chose demeure néanmoins: quels que soient les discours sur la reproductibilité de l'expérience et l'universalité des phénomènes, la traduction technique, parce qu'elle s'adresse elle aussi à un public spécifique, ayant sa propre culture, doit à son tour tenir compte du facteur local. Il ne suffit donc pas de comprendre les phénomènes et de disposer des lexiques adéquats pour les restituer correctement: il faut aussi intégrer l'horizon d'attente des destinataires et savoir de quelle manière, à travers quel prisme, ils perçoivent les phénomènes. C'est en cela, sans doute, que la traduction technique est emblématique du champ pragmatique.

\subsection{Le temporel rejoint le local}

Le progrès des sciences et des techniques constitue un autre phénomène territorialisé. Sa mesure la plus courante (le nombre de brevets déposés à l'échelle nationale) montre qu'il existe des pays plus innovants et d'autres, plus suivistes. Il s'ensuit des décalages temporels dans la prise en compte collective de la nouveauté - avec des répercussions en traduction. Ainsi, dans un texte récent (2008) sur la mesure de l'empreinte carbone, on trouve cette phrase dans l'original anglais:

(2) The definition of "carbon footprint" is surprisingly vague given the growth in the term's use over the past decade.

(Matthews, Hendrickson et Weber 2008 ${ }^{6}$ )

Une analyse de corpus (Zylberberg 2010) montre que le terme carbon footprint, est effectivement attesté depuis 1998 environ (soit dix ans avant la publication de 
l'original). La même analyse montre que l'équivalent français empreinte carbone ne s'impose que depuis plus récemment. Une traduction exacte sur les plans spatial et temporel, quoique moins précise linguistiquement, serait alors:

(3) S'il est de plus en plus question d'empreinte carbone depuis quelques années, la définition de ce concept reste étonnamment vague.

(Zylberberg 2010: 23)

En effet, nous avons en anglais un terme dont l'équivalent français a mis plus de temps à s'imposer: mieux vaut donc déplacer le centre de gravité du texte de ce terme vers le concept qu'il recouvre (en étant bien conscient qu'un terme et une notion ne sont pas une même chose), et être un peu moins précis sur le nombre d'années pour être plus juste sur le plan local. Ici, la terminologie rencontre la traduction et le temporel s'allie au local, avec un effet de rayonnement du centre (les foyers d'innovation, d'où vont également surgir les termes) et de la périphérie. Faute de correctifs de ce type, la culture source pourrait fort bien imposer ses termes, ses concepts et sa temporalité à une culture cible. Le choix de la traductrice a donc pour effet de sauvegarder ce en quoi cette culture cible se distingue de la source, à seule fin de ne pas interrompre la lecture par le destinataire du texte: c'est une préservation qui vise à donner au lecteur l'illusion de la transparence. On peut donc considérer que deux formes de focalisation culturelle (celle de la cible et celle de la source) sont ici en concurrence. Nous sommes donc bien, en traduction technique, dans la situation décrite, dans la sphère littéraire, par Even-Zohar (1978/2000), dans sa théorie du ou des polysystèmes, ou par Venuti lorsqu'il déplore «the ethnocentric violence of translation» (Venuti 1995: 20).

\subsection{Entre impérialisme et relativisme culturels}

Élargissons maintenant notre focale à l'économie. En politique monétaire, il arrive que l'on croise, en anglais, le concept de original sin, qui décrit la situation de pays en développement qui ont besoin, pour renforcer leur monnaie, d'emprunter, mais ne peuvent le faire en pratique qu'en devises (et le plus souvent en dollars), ce qui, à terme, a pour effet d'affaiblir encore plus leur unité de compte. Cette notion riche de ce qu'elle dénote - et surtout de ce qu'elle connote - s'applique en particulier à certains pays latino-américains, et surtout à l'Argentine sur une grande partie de son histoire récente. Elle est due à des chercheurs de la Banque interaméricaine de développement (voir par exemple Eichengreen et Hausmann 1999). Travaillant vers le français, pour un lectorat a priori au fait de son substrat religieux, il est aisé de le traduire littéralement: péché originel. Mais comment aurions-nous fait si nous avions eu à le traduire vers le malais, le chinois, l'arabe ou le japonais?

Traducteur consciencieux - ce qui ne va pas sans une forme d'inconscience quant au temps à consacrer à ce type de questions -, nous aurions pu nous plonger (en traduction, certes) dans le Ramayana ou les œuvres de Confucius, et nous aurions peut-être déniché une expression vaguement équivalente sur le plan culturel. Notre texte y aurait gagné sur le plan de l'équivalence d'effet. Il y aurait par contre perdu en termes de réversibilité et d'unité conceptuelle: qui peut garantir qu'en utilisant la même stratégie pour retraduire le même concept du malais, du hindi ou du japonais vers l'anglais, nous serions retombé sur l'expression original sin? (À ce sujet, voir Zhang, à paraître.) Pour rester dans la même sphère culturelle, nous ne sommes pas 
très loin du chantier de la tour de Babel... En écrivant cela, nous n'affirmons certes pas que la rétrotraduction constitue toujours une démarche pertinente pour juger de la validité d'un travail. Ici, néanmoins, c'est le cas parce qu'elle confère l'assurance que, dans le débat, toutes les parties prenantes parlent bien de la même chose. C'est le théorème de la brioche aux raisins, dû à Danica Seleskovitch (1975: 30, cité par Lavault, à paraître). Le plus simple apparaît donc d'exporter notre référence culturelle judéo-chrétienne dans ces langues portées a priori par une culture autre. Cette démarche se pare néanmoins du doux nom d'impérialisme culturel. Cette question, fort débattue en traduction littéraire, notamment dans son courant postcolonial, ne se pose pas avec moins d'acuité dans le champ pragmatique. Une troisième démarche aurait consisté à neutraliser l'allusion, soit en trouvant une formulation moins connotée culturellement (fragilité monétaire intrinsèque, par exemple), soit en détournant une expression existante (cercle vicieux monétaire...). Est-elle à recommander? Sans doute là où les préoccupations politiques l'imposeraient: en situation réelle, il n'est pas toujours mauvais d'opter pour le politiquement correct. Cependant, c'est bien le seul argument en faveur d'une telle option. En effet, celle-ci induit une perte sur les plans à la fois de la densité de sens et de l'unicité du concept. Comme souvent en traduction, la solution médiane est la plus mauvaise.

En d'autres termes, si j'adapte mon texte aux attentes que je prête à son public cible, j'en facilite la réception, au risque de susciter un malentendu quant au substrat culturel: je procède à une dissémination du sens. Si, en revanche, je conserve ma référence culturelle initiale (et même originelle, ici), je reconnais implicitement qu'il existe, culturellement, un centre qui impose ses valeurs et une périphérie qui est réduite, soit à adopter ces dernières soit à se couper du système de communication. Ce qui conduit à s'échouer sur un autre écueil éthique, celui du relativisme culturel. Enfin, si j'opte pour l'entre-deux, mon texte perdra de son tranchant et donc de sa pertinence. Par conséquent, le facteur local nous contraint, ici, à choisir entre trois solutions imparfaites, comportant chacune des implications communicationnelles ou idéologiques. La traduction n'est pas une activité neutre; elle comporte des implications politiques. Est-il bon d'en avoir conscience? Certainement pour la qualité des traductions et la prise de distance. Pas forcément pour le moral de certains traducteurs, qui peuvent trouver douloureux, voire insupportable d'avoir à restituer avec la plus grande fidélité des écrits et des modes de pensée qui, occasionnellement, sont aux antipodes des leurs. Nous sommes ici au bord du sentiment d'imposture (Froeliger, à paraître).

\subsection{Facteur local et imaginaire social}

«Have FUN along the way! "Sommes-nous, ici, dans le secteur touristique? Ou celui des jeux vidéo? Ni l'un ni l'autre. Cette injonction d'un goût «très sûr » clôt une liste à puces détaillant le protocole à suivre en médecine hospitalière pour évaluer la gravité du risque encouru par des patients souffrant d'escarres (essentiellement) et d'ulcères du pied diabétique. Perplexité de la traductrice à qui ce texte a été confié: l'auteur du texte initial n'a pas trouvé incongru de formuler cette recommandation. N'étant pas psychosociologue, il nous est difficile de dire si elle l'est effectivement pour un lectorat formé de médecins anglophones (du Canada, en l'occurrence ${ }^{7}$ ). Nous en sommes donc réduits à des hypothèses assez fragiles sur la justification dans le 
texte de départ: en première analyse, on pourra penser que, dans la culture du rédacteur d'origine (à moins qu'il ne s'agisse d'un trait purement individuel et propre à l'auteur), il est de bon ton de tout envisager positivement, le sourire aux lèvres. Si c'est de l'humour, ce sera donc très probablement un humour très bon enfant, au premier degré. Ce que nous savons en revanche avec certitude, c'est qu'un médecin francophone (également du Canada) ne pourra qu'être choqué par une telle phrase - tout comme l'a été la traductrice. Là encore, il nous faut localiser: sans avoir de certitudes sur l'imaginaire social qui peut conduire à écrire une telle formule en anglais, nous savons que sa transposition linguistique ne passera pas en français. Car le trait d'humour ne sera plus du tout innocent, mais prendra un tour franchement noir. Nous, traducteurs, ne maîtrisons pas toujours l'imaginaire social qui informe le texte de départ, mais nous savons au moins pour qui nous écrivons et les effets que certaines formulations pourront produire sur ce lectorat. C'est la raison pour laquelle la traductrice confrontée à ce problème a, après consultation du demandeur, décidé de supprimer cette mention: elle n'apportait rien et mettait en péril la réception du reste du message. En revanche, l'original anglais est resté tel quel alors que les deux textes étaient destinés à être publiés simultanément. Justification du responsable de la publication: "Cela dédramatise la situation.» Mais dans une langue seulement...

\subsection{Ce que le texte dit ou ce qu'il fait - et aux yeux de qui?}

Passons maintenant à la presse. Les phrases suivantes ont paru dans l'hebdomadaire Die Zeit à la toute fin décembre 2004, au moment où un terme de marine d'origine japonaise a commencé à devenir synonyme de catastrophe. Elles sont rédigées pour un public germanophone et extraites d'un texte sur, on l'aura compris, les tsunamis:

(4) Im November 1755 war der Atlantik an der Reihe: Nach dem schweren Erdbeben von Lissabon, das Kleist zu seiner Erzählung Das Erdbeben in Chili inspirierte, türmte sich das Wasser bis zu zehn Meter hoch auf.

(Schwelien et Willmann 2004 ${ }^{8}$

Il n'y a guère de difficulté de traduction a priori:

TABLEAU 5

\section{Ce que dit le texte allemand}

En novembre 1755, c'est l'Atlantique qui est frappé: après le tremblement de terre de Lisbonne, qui a inspiré à Henrich von Kleist sa nouvelle «Le tremblement de terre du Chili» (parue en français chez Garnier Flammarion, dans le recueil intitulé La Marquise d'O, 1990). qui est frappé: plutôt que C'était au tour de l'Atlantique, traduction littérale et quelque peu obscène compte tenu de l'ampleur de la catastrophe en question.

tremblement de terre: compte tenu de ce qui va suivre, il est inutile de traduire schwer.

Heinrich von: l'indication du prénom permettra au lecteur français de mieux identifier cet auteur parmi la masse de ceux qui pourraient se trouver dans sa mémoire: le lecteur allemand n'a pas besoin de la même précision.

Référence bibliographique: ajout nécessaire pour raccorder le texte à la réalité pour le lecteur francophone, les vagues ont pu atteindre jusqu'à dix mètres de hauteur (d'après les commentaires des étudiants). 
Voilà globalement - et avec quatre manipulations ou explicitations mineures, justifiées dans la colonne de droite - ce que disent ces phrases, c'est-à-dire leur sens. Mais qui, dans un public francophone, a lu Le tremblement de terre du Chili de Kleist ${ }^{9}$ ? Cette référence culturelle parle au public germanophone et devient muette pour la grande majorité des autres: les mots (la composante dénotative) sont les mêmes, mais les associations (l'aspect connotatif) diffèrent ou font défaut. Nous avons la langue, nous avons la signification; nous n'avons plus l'effet. Je serai donc bien avisé de remplacer Kleist par Voltaire et «Le tremblement de terre du Chili» par "Candide», œuvre qui, cette fois, est familière du public francophone et a été rédigée dans des circonstances analogues. Et je ne procéderai bien sûr à cette substitution que si elle sert bel et bien l'intention de mon client. En effet, s'il s'agit de rendre compte de ce qu'écrit la presse allemande au sujet du tsunami, je garderai la référence initiale. Si, en revanche, l'objectif est d'informer mes lecteurs sur la nature de ces phénomènes catastrophiques, je lui préférerai Voltaire: je localiserai. Cette opération, une fois de plus, est assez banale sur le plan pratique. Néanmoins, une intéressante difficulté surgit si nous cherchons à replacer une telle solution dans un contexte théorique. On nous fera observer, avec justesse, que cet exemple - comme d'ailleurs ceux qui le précèdent - cadre parfaitement avec la théorie fonctionnaliste telle que développée, en particulier, par Nord (1997) à la suite de Vermeer et Reiss. Et l'on pourra alors formuler l'hypothèse que localisation (au sens large) et fonctionnalisme renvoient en fait à une seule et même chose. Dans les deux cas, il s'agit bel et bien d'adapter la traduction à une catégorie de destinataires, en fonction de la nature du texte de départ et de ce qui est attendu du texte d'arrivée (Nord dirait cahier des charges, analyse du texte source, hiérarchisation des problèmes de traduction). Les fonctionnalistes ont-ils pour autant le monopole d'une telle vision? Certainement pas: on pourrait tout aussi bien renvoyer à Nida et Taber (1969/2003), avec la notion d'équivalence dynamique: il faut s'adresser au destinataire en usant du système de référence de ce dernier afin de provoquer chez lui le même stimulus que le texte de départ dans son contexte initial.

Il n'y a donc pas un mais plusieurs voisinages théoriques à l'horizon des pratiques de localisation textuelle. Avec au demeurant une assez grande compatibilité entre ces différentes approches. Mais c'est ici que commence la véritable difficulté: l'injonction à traduire non pas ce que le texte dit mais ce qu'il fait ne se trouve, à notre connaissance, formulée explicitement ni chez les fonctionnalistes ni chez Nida et Taber, mais chez Meschonnic, et cela à de multiples reprises (voir notamment Meschonnic 1999: 22, 55, 124 et 139, ou encore Meschonnic 2006: 132: «plus que ce qu'un texte dit, c'est ce qu'il fait qui est à traduire; plus que le sens, c'est la force, l'affect »). Or, nous nous trouvons là sur un tout autre continent traductologique. Et pourtant, c'est Meschonnic qui dit avec le plus de force qu'une traduction de qualité est d'abord un texte qui puisse à son tour prétendre au statut d'original. Et c'est bien ce à quoi une démarche de localisation peut prétendre... Il est donc frappant que des courants aussi opposés se rejoignent (théoriquement, car les solutions proposées seraient sans doute antithétiques si nous partions non pas de la pratique pour aller à la théorie, mais de la perspective inverse) sur ce point du facteur local. Comment tenter de dépasser cette contradiction, et le faut-il? 


\subsection{Une vaine tentative de conciliation}

Il paraît tentant, en première analyse, de réfléchir à l'énigme de cette ressemblance dans la dissemblance sans quitter pour l'instant le cadre traductologique envisagé depuis le début de cet article. Deux options nous paraissent alors possibles: chercher des points communs à ces deux positions, ou tenter de greffer l'une sur l'autre.

En ce qui concerne les points communs, il est tout d'abord aisé de constater que l'ensemble des auteurs considérés prennent leurs distances vis-à-vis de la linguistique (en tout cas dans la vision codique de cette dernière) : c'est un terrain d'entente indiscutable de tout théoricien et de tout praticien cherchant à prendre en compte le facteur local. Il faut ensuite observer que Nord, Nida et Taber, ainsi que Meschonnic, sont tous traducteurs de la Bible et consacrent au moins une partie de leur réflexion à la traduction de cet ouvrage. Et c'est peut-être ce qui explique l'impasse théorique dans laquelle nous nous trouvons. Ce point commun, qui pourrait les rapprocher, les sépare radicalement, sur le modèle d'un aphorisme faussement attribué à Oscar Wilde, et qui voit dans l'Angleterre et les États-Unis deux pays séparés par une langue commune. Ici, c'est le même livre qui divise nos auteurs. En effet, pour Nida et Taber, qui s'adressent au départ à des traducteurs chargés de diffuser le message biblique auprès de populations autochtones et indigènes, il s'agit clairement d'imposer une vision du monde (une métaphysique) nouvelle par des formulations rendues pour cela familières. D’où la désormais classique conclusion de ces derniers:

Perhaps no better compliment could come to a translator than to have someone say, "I never knew before that God spoke my language." (Nida et Taber 1969/2003: 173)

Christiane Nord, pour sa part, propose de traduire l'Évangile de Jean selon une optique que nous rapprocherions des stratégies employées en publicité:

If you want to attract people to your cause you would probably not start by telling them that it is not worth the effort in the first place. [...] We interpreted the text in the light of its own situation, and translated for an audience in a different situation precisely to make sure that the sense we elicited was ferried to the other shore. (Nord 2006: 57)

Dans les deux cas, nous sommes dans une démarche d'évangélisation. Rien de tel chez Meschonnic, qui s'attache à restituer «la force $d u$ verbe» (allusion à Cicéron; Meschonnic 2006: 127) et la rugosité du texte original: "Il y a une sauvagerie, et violence sur violence, dans cette histoire. Et elle passe par une force du langage, y compris des violences syntaxiques» (Meschonnic 2002: 9). Phrase dans laquelle on perçoit d'ailleurs l'écho du Georges Mounin des Belles infidèles: «[...] quand la Bible aura trouvé son Leconte de Lisle, on lira sans doute avec des yeux stupéfaits des documents ethnographiques inconnus ou presque» (Mounin 1955: 138). L'enjeu est le même: un texte efficace, comme partout en traduction; les présupposés n'ont rien de commun. L'accord est donc minimal et accidentel.

Peut-on alors tenter de faire annexer l'une de ces théories (celle de Meschonnic, en l'occurrence) par les autres? Il faudrait pour cela une certaine dose de mauvaise foi - c'est le cas de le dire. On pourrait en effet affirmer que la différence essentielle entre les approches considérées tient à la fonction attribuée au texte: prosélyte (c'està-dire religieuse) dans un cas, sacrée et divine dans l'autre (sur ce point, voir Meschonnic 2006: 138): textes équivalents, fonctions différentes, en somme, ce qui ferait de Meschonnic un fonctionnaliste qui s'ignore... Nous n'évoquons toutefois 
cet argument que pour relever son caractère spécieux: il ne permet pas d'assurer une véritable compatibilité des théories envisagées. On pourrait au demeurant retourner ce raisonnement pour se demander si les théories défendues par Nida et Taber ou par Nord sont réellement appropriées à la traduction de textes fondateurs... Plus que dans une querelle traductologique, nous sommes ici dans une controverse idéologique (au demeurant représentable en termes topologiques, c'est-à-dire spatiaux). Et nous n'en sortirons pas tant que nous chercherons à fonder une traductologie valable dans le domaine pragmatique sur des théories établies initialement dans le domaine littéraire. La véritable question à laquelle nous invite la prise en compte du facteur local en traduction n'est donc pas lorsque des auteurs par ailleurs inconciliables s'accordent pour recommander de traduire non pas ce que le texte dit, mais ce qu'il fait, disent-ils oui ou non la même chose? (quel que soit l'intérêt de cette interrogation par ailleurs), mais bien quel horizon théorique envisager pour la traduction pragmatique à partir de nos réflexions sur le facteur local?

\section{Situer la traductologie pragmatique}

Les conclusions traductologiques auxquelles nous arrivons à ce stade sont donc finalement peu novatrices, et se ramènent à trois points. Premièrement, la traduction doit être envisagée comme un phénomène culturel, c'est-à-dire contingent, selon les cas, au temps ou (dans le cas qui nous intéresse) à l'espace. Il n'y a donc pas de correspondances naturelles - on pourrait aussi dire naïves - entre un texte original et sa traduction, mais des choix qui procèdent de facteurs individuels ou sociologiques et que la traductologie se doit de mettre au jour. Deuxièmement, on ne traduit pas des mots mais du discours, c'est-à-dire des textes présentant une structure et une épaisseur (ce qui, là non plus, n'est pas nouveau, et constitue même un des points communs entre Ladmiral et Meschonnic). Non pas de la langue, mais des propositions, qui procèdent de l'interprétation d'une intention (voir à ce sujet Reboul et Moeschler 1998: 89-90). Troisièmement, il importe de ramener l'apport de la linguistique à une proportion raisonnable. Il y a quelques années, beaucoup de linguistes affirmaient que cette science pouvait rendre compte de la totalité des problèmes posés par la traduction. Dans le même temps, beaucoup de traducteurs proclamaient exactement l'inverse. Les points de vue se sont légèrement rapprochés entre-temps, avec une interrogation qui demeure sur la relation appropriée, c'est-à-dire la plus intellectuellement productive pour la pratique et pour la recherche, entre ces deux domaines du savoir. Mais est-ce là tout ce que l'on peut tirer d'une réflexion sur le facteur local en traduction pragmatique - quelques lieux communs largement explorés en traduction littéraire et par la théorie fonctionnaliste, ainsi qu'un point d'accord paradoxal entre théories contradictoires? Certes non. Quelque chose d'important, de spécifique au domaine pragmatique et de lié au local nous échappe encore. Notre hypothèse est que cette chose permettra peut-être d'assigner un lieu plus précis à la traduction pragmatique dans le champ général de la recherche. Nous pensons que cet élément essentiel et manquant est ce que l'on appelle le réel ou le référent - qui ne peut, par définition, pas être traité de la même manière dans les textes de fiction ou de métaphysique...

La mise à l'épreuve de cette hypothèse nous amènera d'abord à nous demander s'il existe des différences de traitement du local entre traductions littéraire et pragma- 
tique, pour revenir ensuite sur ce que le facteur local pourrait dévoiler spécifiquement en traduction pragmatique et enfin nous demander comment situer la traductologie pragmatique d'une manière efficace.

\subsection{Bref détour par la traduction littéraire}

Commençons par observer que la littérature est tout aussi concernée par une représentation des subtilités et des enjeux de la traduction par le local (ce qui est parfaitement normal puisque tous les théoriciens sur lesquels nous nous sommes appuyé tirent de ce champ tout ou partie de leur réflexion). Deux exemples succincts le montreront. Lewis Carroll dans sa préface à la première traduction d'Alice in Wonderland, dont il avait d'ailleurs choisi lui-même le premier traducteur, Henri Bué (premier d'une longue série), remerciait celui-ci d'avoir

[...] remplacé par des parodies de sa composition quelques parodies de morceaux de poésie anglaise qui n'avaient de valeur que pour des enfants anglais, et aussi de ce qu'il a su donner en jeux de mots français les équivalents des jeux de mots anglais dont la traduction n'était pas possible. (Houssin, 2009: 1, $4^{10}$ )

C'est l'équivalent de notre exemple du point 1.5: Kleist est remplacé par Voltaire.

Comme en traduction audiovisuelle (voir l'article de Minguant dans ce numéro), on rencontre un problème du même ordre lorsque le texte à traduire met en scène la langue étrangère vers laquelle doit s'effectuer la traduction. Ainsi, chez Beckett, la réplique suivante de En attendant Godot:

(5) Estragon: (Accent anglais). Oh très bon. Très très très bon.

(Beckett 1952: 53'11)

devient en anglais:

(6) Estragon: Oh, tray bong, tray tray tray bong.

(Beckett 1956: 395 $\left.5^{12}\right)$

Ce serait l'équivalent de l'option relativiste mentionnée au point 1.2 (original

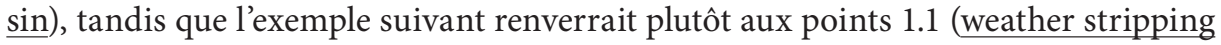
helps) ou 1.4 (Have FUN along the way). Dans ce cas précis, une phrase également à cheval sur deux langues sera carrément omise de la version en anglais:

(7) Estragon: (avec volupté). - Calme... Calme... (Rêveusement). Les Anglais disent câââm. Ce sont des gens câââms.

(Beckett 1952: 2013)

Y a-t-il équivalence, s'agit-il de la même pièce, nous trouvons-nous dans une perspective benjaminienne, dans laquelle le texte ultime, à venir, serait une sorte de projection métaphysique de ses versions terrestres en anglais et en français? Passionnantes questions (et chaudement débattues), qui ne sont pas les nôtres. Contentons-nous d'observer que Samuel Beckett, ici comme ailleurs, est son propre traducteur, ce qui l'autorise à prendre maintes libertés et amène certains critiques à affirmer qu'il n'y a pas réécriture, mais double statut d'original (c'est la thèse de Montini 2007) - ce qui ne facilite d'ailleurs pas les tentatives de traduction dans une langue tierce.

Il n'entre pas dans notre propos de multiplier les exemples de ce type, mais plus modestement de montrer qu'avec ses fins et ses moyens propres, la traduction des 
textes littéraires est bel et bien soumise à la même problématique du facteur local. Et nous renvoyons ici à la théorie du polysystème, déjà évoquée. Cette traduction littéraire va-t-elle pour autant résoudre les mêmes problèmes de manière analogue à la traduction pragmatique? En d'autres termes, les ressemblances ne seraient-elles pas, là encore, trompeuses? Inversons la problématique: si nous avions, dans la première partie de cet article, été face à des textes littéraires et non pragmatiques, comment aurions-nous traité certains des exemples envisagés? Certainement pas de la même manière. Il serait incidemment très intéressant de confronter la traduction de sujets techniques traités dans des textes à visée technique et dans la littérature (dans, par exemple, les romans de William Gaddis, Thomas Pynchon ou Hermann Broch, selon le principe, dû à Bakhtine [1978], que la littérature est la forme d'expression créatrice qui est susceptible d'accueillir toutes les autres formes), mais c'est encore un autre sujet. Nous aurions donc fait ressortir l'étrangeté, l'inhabituel, le remarquable: tout ce qui est susceptible d'arrêter la lecture, afin que le destinataire puisse se dire il y a là un style, une forme spécifique, qui font l'originalité et la valeur du texte. Au contraire, dans tous les exemples mentionnés jusqu'ici, nous avons modifié le texte dès lors qu'une simple transposition de celui-ci pouvait nous sembler choquante ou inappropriée, c'est-à-dire, en l'occurrence, de nature à freiner le cours de la lecture ou de la compréhension (c'est la prétention au classicisme stylistique envisagé par Ladmiral dans ses Théorèmes pour la traduction, 1979). En traduction pragmatique, la prise en compte du facteur local amène ainsi à gommer ou à rendre familier: il s'agit de recomposer tout ce qui peut entraver la lecture, afin de donner au destinataire l'illusion de la transparence. Le fait que les moyens employés soient parfois les mêmes est donc finalement aussi accidentel que la coïncidence observée plus haut sur ce que le texte dit et ce qu'il fait.

\subsection{Existe-t-il une spécificité de la traduction pragmatique?}

Nous avons vu que le local était le lieu d'une anomalie par rapport au linguistique. Mais en pragmatique, cette anomalie a précisément pour objectif de n'en pas paraître une, alors qu'en littéraire, les options sont bien plus nombreuses: on peut user de stratégies diamétralement opposées face à un texte littéraire; c'est beaucoup moins vrai en traduction pragmatique. Le problème auquel nous nous heurtons depuis quelques pages tient en outre à la nature de la littérature tout court, autant qu'à la diversité de la traduction littéraire. En effet, la littérature est création, elle met le monde en mouvement, tandis que la traduction (en général) est simplement la réexpression d'une vision du monde unique (en traduction littéraire) ou partagée (en traduction pragmatique). Mais comment réexprimer l'unique? C’est bien la difficulté à laquelle est confrontée la traduction littéraire, et qui ne se pose pas en pragmatique. Celle-ci n'a pas pour ambition de créer un monde. Elle est confrontée non pas à la fiction, mais au réel: elle se situe du côté du référent (Froeliger 2004). C’est une chance immense, en même temps qu'une obligation de se constituer une culture générale et spécialisée. Et c'est ce référent qui nous indiquera où localiser. Quels points du texte (autre question spatiale) sont concernés? Grave question, si l'on pense aux progrès actuels et prévisibles de la traduction automatique. En effet, elle pourrait, à terme, déterminer les passages justifiant une intervention humaine directe à l'heure où les techniques de post-édition se font de plus en plus efficaces (d'aucuns diraient mena- 
çantes). Ces points sont de deux ordres: les jeux sur la langue, c'est un sujet trop rebattu pour que nous nous y arrêtions, et ce que l'on appelle, là encore, dans les études littéraires, les effets de réel, c'est-à-dire les renvois à des références culturelles. Où donc localise-t-on? On localise dans un imaginaire et dans le réel. Or, on sait que le réel est par essence étrange, incongru, bizarre, inattendu: c'est le lieu de la surprise. La réalité débordant toujours la fiction (il suffit de suivre l'actualité judiciaire de n'importe quel pays pour s'en convaincre), ce qu'on appelle effet de réel, on pourrait donc aussi bien, en traductologie pragmatique, l'appeler effet d'étrangeté. Et c'est précisément en réaction à cette étrangeté du réel qu'en traduction pragmatique, on aura tendance à en rajouter dans le sens de la normalisation, au risque de raboter notre texte (Froeliger 2008). Si la composante locale intervient dans l'opération, c'est qu'il y a une résistance, ancrée dans un territoire et une culture, à ce que l'on pourrait qualifier de traduction pure et simple. Du fait de cet arrière-plan culturel, la justesse d'une traduction va donc parfois tenir à la non-coïncidence d'un objet (le texte traduit) avec lui-même (son original): du point de vue phénoménologique, on peut finalement dire qu'il y a véritablement traduction là où il y a défaut d'identité. Et c'est en pragmatique que ce phénomène apparaît le plus clairement. Passé un certain stade, apprendre à traduire, cela devient ainsi apprendre à dire autre chose que l'original. Ce qui mérite l'attention de la recherche en pragmatique, ce n'est donc pas lorsque deux ou plusieurs textes sont une réplique l'un de l'autre, mais bien lorsqu'ils diffèrent sans diverger. À l'arrivée, néanmoins, le texte se devra de refléter une forme de normalité aux yeux de ses lecteurs. L'effet d'étrangeté doit donc être identifié, analysé, reconfiguré et renormalisé. L'ensemble de cette problématique pourrait bien être métaphorisée dans In the Cage, l'une des nouvelles les plus étranges d'Henry James, dans laquelle une jeune télégraphiste à l'esprit romanesque croit se retrouver, par la seule vertu des messages qu'elle transmet, au cœur d'une intrigue à mi-chemin entre l'espionnage et le roman d'amour:

(8) She was left confronted with her habitual critics. "If it's wrong it's all right!" she extravagantly quoted to them.

(James 1898: $210^{14}$ )

\subsection{Le pragmatique peut-il s'émanciper du littéraire?}

Nous avons évoqué plus haut une dichotomie entre littéraire et pragmatique. Celle-ci ne se manifeste pas que sur le plan théorique: on l'observe aussi dans le fonctionnement de l'institution (nous entendons par là l'université, beaucoup de revues spécialisées et la presse), qui a tôt fait de distinguer entre ce qui lui apparaît comme noble (les grands textes, la littérature) et ce qu'elle considérera comme vulgaire (la culture de masse, la technique, les textes à visée communicationnelle: la parole creuse, auraient dit certains). Ce schéma, répétons-le, ne nous convient plus, à l'heure, notamment, où le centre de gravité en recherche se déplace, se déterritorialise, «comme des petites colonnes de fourmis » (Deleuze et Guattari 1980: 33), des textes vers ceux qui les produisent (voir Froeliger [2005] ou Chesterman [2009], par exemple). Est-il normal, est-il sain, est-il souhaitable que le gros de la recherche en traduction se concentre encore dans la sphère littéraire alors que la grande majorité des traductions s'effectuent dans le domaine pragmatique? Non. 
En effet, nous l'avons dit, ces textes pragmatiques soulèvent globalement des problèmes tout aussi passionnants - et passionnants parce que bien souvent différents dans leurs enjeux ou dans leurs solutions. En outre, ce découpage binaire fait l'impasse sur tout ce qui se situe entre ces deux extrêmes, ce que Giancarlo Marchesini (2009) appelle les testi medi, ou textes mixtes, ou encore intermédiaires, c'est-à-dire ni complètement littéraires ni exclusivement communicationnels ${ }^{15}$. On le voit bien avec la traduction des sciences humaines: les pragmatiques d'un côté, les littéraires de l'autre considèrent que celle-ci est leur exclusive affaire. Avec à la clé de nouvelles querelles de territoires: à l'université, en France, les premiers se rencontrent en langues étrangères appliquées (LEA), et les seconds dans les UFR (unités de formation et de recherche) de langues, littératures et civilisations étrangères (LLCE). Et il n'est pas rare d'entendre poser, en toute honnêteté, en toute innocence, avec à la rigueur un zeste de condescendance, par des collègues littéraires cette question toute simple: "Alors on fait aussi de la recherche en $L E A^{16}$ ?"

Autre difficulté de positionnement à l'université, la traductologie pragmatique se trouve en porte à faux avec le traditionnel découpage qui veut que l'on recrute d'abord un spécialiste d'une langue (ce qui correspond, en France, aux sections du Conseil national des universités, ou CNU) et ensuite, soit un linguiste soit un civilisationniste. Or, les praticiens de la traduction se considèrent, eux, comme des spécialistes de la traduction plutôt que de la langue. Et à tout prendre, justement, les problèmes qui font leur quotidien touchent beaucoup plus souvent à l'organisation du référent (à la civilisation, donc) - observation qui laisse au demeurant quelque peu sceptiques leurs collègues estampillés civilisationnistes... C'est une nouvelle illustration du caractère interstitiel de la traduction et de la traductologie (voir McCarty 1999). La traductologie pragmatique est-elle alors condamnée à rester dans les marges, ou ne peut-on imaginer une cartographie plus appropriée à une recherche fructueuse dans ce domaine? Cela suppose, à notre sens, un double mouvement: d'émancipation, d'une part, de redéfinition des voisinages, d'autre part.

Partout ailleurs, le mouvement vers une théorie procède par élimination et élagage de l'objet, en mettant de côté tout ce qui est annexe, a priori improductif ou relevant d'une discipline autre. C'est ainsi que la Société de linguistique de Paris a, en 1866, interdit, dans son cénacle, les discussions sur l'origine du langage, "parce qu'elle y voyait l'occasion de débats purement spéculatifs et stériles» (Reboul et Moeschler 1998: 14). C'est ainsi que Witold Gombrowicz (1969) a pu écrire, ou plus exactement dicter, un ouvrage intitulé Cours de philosophie en six heures et quart, au motif que, pour cet auteur, toutes les questions de la philosophie convergent sur l'existentialisme et qu'on peut faire le tour de cette école en quelques heures à peine (affirmation que nous nous contenterons lâchement de rapporter sans chercher à la juger ni à la jauger)... Ce premier mouvement est nécessaire pour donner à la traductologie pragmatique une autonomie qui la rende plus cohérente et plus efficace. C'est ce que nous avons tenté de faire dans les pages qui précèdent.

Mais pour répondre à l'interrogation sous-jacente à toute science (qu'est-ce qu'une question pertinente dans le domaine considéré?), il faut aussi ouvrir le champ. La traduction pragmatique, rappelons-le, consiste à lever les malentendus (Froeliger 2006) pour arriver à ce que des individus et des groupes se parlent et se comprennent (ce qui n'est pas la même chose). Et la recherche dans ce secteur, si elle veut être utile et productive, se doit d'adhérer à ce modèle, en donnant la parole à la linguistique, 
à la civilisation, à la communication, à la littérature en tant que telle, à l'ingénierie des langues, à l'histoire, à la philosophie, aux sciences cognitives, etc., mais en tant que disciplines voisines et non comme modèles dominants. Sans se faire annexer par aucune d'entre elles, elle peut faire son miel de chacune: «interdiscipline» (voir Snell-Hornby, Pöchhacker et al. 1994) n'est pas obligatoirement synonyme d'interstitialité. Pour que le brassage soit fécond, il importe simplement de remplir deux critères, là encore représentables en termes territoriaux. En effet, il faut un dialogue entre égaux, sans tentations impérialistes d'un domaine sur l'autre. Dans le même temps, il faut que ceux dont l'activité principale constitue le cœur de nos réflexions - nous parlons des traducteurs actuels et en devenir - puissent y trouver leur compte: la réflexion, quels que soient ses sources et ses trajets, doit être rapportée à la pratique.

La recherche appliquée n'a donc pas à se sentir en situation de minorité par rapport à la recherche fondamentale, pas plus que le pragmatique ne doit avoir de complexes vis-à-vis du littéraire. Dans un cas comme dans l'autre, la réflexion sur le local peut constituer un moyen privilégié d'établir une cartographie favorisant un agencement adéquat entre ces différents et complémentaires domaines, avec comme horizon la pondéreuse question de l'unité des sciences de la culture ${ }^{17}$. La traduction littéraire constitue un champ distinct, qui ne saurait ni s'imposer ni s'opposer terme à terme à la traduction technique, de presse, juridique, informatique, audiovisuelle, économique, etc. Les problèmes sont généralement du même ordre. Les solutions, en revanche, seront souvent différentes parce que la visée (l'intention) des textes n'est pas la même (ce qui nous ramène d'ailleurs dans les parages de la théorie fonctionnaliste). Et c'est une des raisons qui rendent féconde la confrontation. Est-ce à dire qu'il ne faudrait plus lire les théoriciens de la traduction littéraire lorsqu'on s'attache au domaine pragmatique. Bien sûr que non! Une culture générale de la traduction est de toute manière indispensable. Ne serait-ce que pour faire ressortir l'historicité des pratiques qui nous semblent les plus naturelles simplement parce que ce sont celles de notre époque et de notre domaine de traduction. Ne serait-ce que pour prendre conscience des enjeux idéologiques de ce que nous écrivons. Ne serait-ce que parce que certaines des règles de fonctionnement de la traduction pragmatique ont été mises en lumière avec une très grande pertinence par des chercheurs ayant fondé toute leur réflexion sur le littéraire, comme Berman ou Venuti, tous deux déjà cités. En effet, une grande partie de ce que ces chercheurs reprochent à ce qu'ils appellent (à tort ou à raison) les mauvais traducteurs littéraires correspond exactement à ce qu'il importe de faire pour produire une traduction pragmatique de qualité. Mais il est tout de même complexe de lire leurs textes à rebours, comme des théories négatives de la traduction pragmatique et il n'est pas certain qu'ils en seraient ravis. Faire de cette dernière - et de la recherche qui la sous-tend - un champ de plein droit de la traductologie donnerait un peu de positivité à cet ensemble.

\section{REMERCIEMENTS}

Nous remercions Camille Promérat, Chiara Montini, Clara Zylberberg, Marie Gravey, Mélissa Médart et André Chassigneux pour leurs exemples et leurs conseils. 


\section{NOTES}

* UFR EILA, Laboratoire CLILLAC-ARP (équipe d'accueil 3967).

1. Comme l'a montré Lyliane Szjnader lors d'un colloque dont les actes paraîtront prochainement (Szjnader, à paraître).

2. Voir l'article Grandeur sans dimension dans Wikipédia. Consultée le 9 septembre 2010, <http:// fr.wikipedia.org/wiki/Grandeur_sans_dimension pour ces grandeurs $>$.

3. CertainTeed Corporation (2002) Noise Control in Buildings, Guidelines for Acoustical ProblemSolving. Consultée le 9 septembre 2010, <http://files.buildsite.com/dbderived-f/certainteed/ derived_files/derivedC376884.pdf >. Cité et traduit dans Promerat (2009) : 33-36.

4. Certains ont au demeurant décidé de satisfaire aux deux exigences. C'est le cas de Sony: voir, par exemple, leur page Web sur le format des téléviseurs. Consultée le 8 août 2010, <http://www.sony. $\mathrm{fr} / \mathrm{lang} / \mathrm{fr} / \mathrm{hub} /$ televiseurs-lcd-bravia/4/1>.

5. Le donneur d'ouvrage a autorisé la citation de cet exemple.

6. Matthews, Scott H., Hendrickson, Chris T. et Weber, Christopher L. (2008): The Importance of Carbon Footprint Estimation Boundaries. Environmental Science and Technology, 42(16) : 58395842. Date de publication: 13 août 2008. Consultée le 9 septembre 2010, <http://pubs.acs.org/doi/ $\mathrm{pdf} / 10.1021 / \mathrm{es} 703112 \mathrm{w}>$.

7. Le donneur d'ouvrage a autorisé la citation de cet exemple.

8. Schwelien, Michael et Willmann, Urs (31 décembre 2004): Ohne jede Warnung - Eine Schockwelle rast vom Indischen Ozean um die Erde. Die Chronik einer Zerstörung. Die Zeit, Hambourg, Allemagne. Consulté le 4 février 2010, <http://www.zeit.de/2005/01/Haupttext >.

9. Kleist, Henrich von (1807): Das Erdbeben in Chili. Texte original republié en ligne, consulté le 3 février 2010, <http://gutenberg.spiegel.de/ ?id=5\&xid=1463\&kapitel=1\#gb_found $>$. Version française: KLEIST, Henrich von (1990): Le tremblement de terre du Chili. In: La Marquise d'O (sans mention de traducteur). Paris: Garnier Flammarion.

10. Houssin, Xavier (17 juillet 2009): Quand Alice est vraiment au pays des merveilles. Le Monde des livres [supplément au quotidien Le Monde], Paris, 1 et 4.

11. Beckett, Samuel (1952): En Attendant Godot. Paris: Les Éditions de Minuit.

12. Beскетt, Samuel (1956): Waiting for Godot - A Tragicomedy on Two Acts. Londres: Faber and Faber.

13. Beckett, Samuel (1952): En Attendant Godot. Paris: Les Éditions de Minuit.

14. James, Henry (1898/1982): In the Cage. In Selected Tales. Londres: Everyman's Library.

15. Il est à noter que la question de la typologie (avec un choix entre le binaire et le ternaire, et leurs raffinements) est aussi traitée longuement dans Ladmiral (1981) et Reiss (2009).

16. Signalons à cet égard qu'il existe depuis peu une collection dédiée à ce champ spécifique du savoir, et baptisée Travaux interdisciplinaires et plurilingues en Langues Étrangères Appliquées, sous la direction de Klaus Morgenroth, Paul Vaiss et Joseph Farré, et publiée par les universités de Paris X Nanterre et Grenoble 3.

17. Nous devons cette observation à François Rastier, lors de la discussion qui a suivi son intervention au colloque La traduction: philosophie, linguistique et didactique, organisé à Villeneuve d'Ascq du $1^{\text {er }}$ au 3 avril 2009, sous la direction de Tatiana Milliaresi.

\section{RÉFÉRENCES}

Bakhtine, Mikhaïl (1978): Esthétique et théorie du roman. Paris: Gallimard.

Berman, Antoine (1999): La traduction et la lettre ou l'auberge du lointain. Paris: Éditions du Seuil.

Cancio, Carmelo et Belmonte, Sydney (2010): De la localisation à la délocalisation: enjeux professionnels. In: Nicolas Froeliger et Jean-René Ladmiral, dir. Meta. 55(4):661-673.

Chesterman, Andrew (2009): The Name and Nature of Translator Studies. Hermes, Journal of Language and Communication Studies. 42:13-22.

Deleuze, Gilles et Guattari, Félix (1980): Mille plateaux. Paris: Éditions de Minuit.

Eichengreen, Barry et Hausmann, Ricardo (1999): Exchange Rates and Financial Fragility. NBER Working Paper 7418. Consultée le 10 septembre 2010, <http://citeseerx.ist.psu.edu/ viewdoc/download?doi=10.1.1.37.2097\&rep=rep1\&type=pdf $>$. 
Even-Zohar, Itamar (1978/2000): The Position of Translated Literature within the Literary Polysystem. In: Lawrence Venuti, dir. (2000): The Translation Studies Reader. Londres/ New York: Routledge, 192-197.

Froeliger, Nicolas (2004): Felix culpa: congruence et neutralité dans la traduction des textes de réalité. Meta. 49(2):236-246.

Froeliger, Nicolas (2005): Placer le traducteur au cœur de la traductologie. In: André Clas, dir. Pour une traductologie proactive, Meta. 50(4) (sur CR-ROM).

Froeliger, Nicolas (2006): Dompter le malentendu: les tâches de la traduction professionnelle. Traduire. 211:59-71.

Froeliger, Nicolas, dir. (2008): Traduction et normalisation. Perros-Guirec: Tribune internationale des langues vivantes, 45.

Froeliger, Nicolas (à paraître) : Le sentiment d'imposture en traduction. In: Florence LAUTeLRipstein, Jean-Yves Masson et Gius Gargiolo, dir. Actes du colloque international Jean-René Ladmiral: une cuvre en mouvement. (Paris: La Sorbonne, Paris IV, 3-5 juin 2010).

Gombrowicz, Witold (1969): Cours de philosophie en six heures et quart. Paris: Rivages.

LAdMiral, Jean-René (1979): Traduire: Théorèmes pour la traduction. Paris: Payot.

LAdMIRAL, Jean-René (1981): Éléments de traduction philosophique. Langue française. 51:19-34.

LAVAULT, Elisabeth (à paraître): L'art de la formule en pédagogie de la traduction. In: Florence Lautel-Ripstein, Jean-Yves Masson et Gius Gargiolo, éd. Actes du colloque international Jean-René Ladmiral: une œuvre en mouvement. (Paris: La Sorbonne, Paris IV, 3-5 juin 2010).

Marchesini, Giancarlo (2009): Per una tipologia traduttiva dei testi medi. In: Vito Pecoraro, dir. Atti del convegno (Giornate internazionali du studi sulla traduzione/Journées internationales d'études sur la traduction, Cefalù, 30-31 octobre et $1^{\text {er }}$ novembre 2008). Vol. II. Palerme (Italie), Studi Francesi, Herbita Editrice: 191-206.

McCarty, Willard (1999): Humanities Computing as Interdiscipline. Consultée le 18 mars 2010, $<$ http://www.iath.virginia.edu/hcs/mccarty.html $>$.

Meschonnic, Henri (1999): Poétique du traduire. Paris: Verdier.

Meschonnic, Henri (2002): Au commencement. Traduction de la Genèse. Paris: Desclée de Brouwer.

Meschonnic, Henri (2006): Le sens du langage. In: Marianne Lederer, dir. Le sens en traduction. Caen: Lettres modernes Minard, Cahiers Champollion. 10:127-139.

Minguant, Nolwenn (à paraître): Commerce, politique et identité: Les enjeux du doublage et sous-titrage des films hollywoodiens. (Actes du colloque Commerces et traduction - Pour une approche pluridisciplinaire et plurilingue des jeux et enjeux de la traduction, Université Paris X, Nanterre, 27-29 novembre 2008).

Montini, Chiara (2007): La bataille du soliloque. Genèse de la poétique bilingue de Samuel Beckett (1929-1946). Amsterdam/New York: Rodopi.

Mounin, Georges (1955): Les belles infidèles. Paris: Éditions des cahiers du Sud.

NidA, Eugene A. et TABER, Charles R. (1969/2003): The Theory and Practice of Translation. Leyde: E. J. Brill.

Nord, Christiane (1997): Translating as a Purposeful Activity: Functionalist Approaches Explained. Manchester: Saint Jerome.

Nord, Christiane (2006): Ferrying Sense over Wide Waters, Skopos Oriented Translation of Biblical Texts. In: Marianne Lederer, dir. Le sens en traduction. Caen: Lettres modernes Minard, Cahiers Champollion. 10:51-62.

Reboul, Anne et Moeschler, Jacques (1998): La pragmatique aujourd'hui - Une nouvelle science de la communication. Paris: Points Essais.

Reiss, Katharina (2009) : Problématiques de la traduction. (Traduit de l'allemand par Catherine BocQueT). Paris: Anthropos/Economica.

Seleskovitch, Danica (1975): Language, langues et mémoire. Paris: Minard.

Snell-Hornby, Mary, Pöchhacker, Franz et Kainde, Klaus, dir. (1994): Translation Studies as an Interdiscipline. Amsterdam/Philadelphie: John Benjamins. 
Szjnader, Lyliane (à paraître): De la Bible hébraïque à la Vulgate: ce que traduisent les choix lexicaux de St Jérôme. In: Tatiana Miliaresi, dir.: La traduction: philosophie, linguistique et didactique. Actes du colloque du même nom, Villeneuve d'Ascq, ${ }^{\text {er }}-3$ avril 2009.

Venuti, Lawrence (1995): The Translator's Invisibility: A History of Translation. Londres/New York: Routledge.

ZHANG, Yinde (à paraître) : Traduction français/chinois en sciences sociales: pratiques et savoirfaire, contribution au colloque international. Actes du colloque Commerce et traduction: pour une approche pluridisciplinaire et plurilingue des jeux et enjeux de la traduction. (Université de Nanterre Paris X, 27-29 novembre 2008).

Zylberberg, Clara (2010): Carbon Footprinting/Le calcul de l'empreinte carbone. Mémoire de master professionnel en traduction non publié. Paris: ILTS, Université Paris Diderot. 